\title{
Mammalian cells put on a display
}

Using a chemokine receptor, researchers at Stanford University have generated a new system to display random peptide libraries on the surface of mammalian cells.

Peptide display, the screening of random peptide libraries to find a motif that interacts with a biomolecule of interest, is a powerful technique in the molecular biologist's toolbox. By far the most popular scaffolds for display experiments are prokaryotic phages. Yet for some applications, the absence of eukaryotic post-translational modification on phage-displayed peptides is a serious drawback. Researchers have thus experimented with alternative eukaryotic systems, such as displaying peptides on the surface of yeast or of baculovirus, which is produced in insect cells.

But when one is looking at peptides that are normally expressed on the surface of mammalian cells, the context of a real mammalian membrane is irreplaceable. As Garry
Nolan of Stanford University puts it, "There are situations where you are coming at an epitope, and you want it to be as much as possible in physiologic context, where it got the appropriate secondary modifications of sugar or other things in the normal surface of the cell, such as lipid components, that you are just not going to find in yeast". In a recent paper in the Journal of Biological Chemistry, Roland Wolkowicz, a research associate in Nolan's lab, presents a new system to display peptide libraries on the surface of mammalian cells.

For any display system, the key to success is a scaffold that will be expressed at a high level and will display the peptide in a position that maximizes exposure. For this purpose, Wolkowicz and Nolan were inspired by the results of Warner Greene's group at the University of California, San Francisco, who showed that a specific epitope could be recognized in the context of the chemokine

\section{A 'FLAKY' BUT USEFUL FLUOROPHORE}

Detecting small changes in protein conformation in living cells would seem to be a daunting task; however, a new fluorescent compound with substantial advantages over previous fluorophores promises to make this easier.

Everyone is familiar with the way the environment you find yourself in can influence your actions, and with the fact that some people are more susceptible to this than others. As it happens, the same is true for fluorescent molecules. Generally the most desirable fluorophores are those that are influenced very little by their environment and function within strict limits. However, in some cases such stability is undesirable and you want a fluorophore that will just 'flake out' and change the way it absorbs and emits light when its environment changes. For example, researchers have exploited the intrinsic fluorescence and environmental sensitivity of the amino acid tryptophan to detect changes in protein conformation. They found that a conformational change can alter the local environment of tryptophans in a protein and cause a measurable change in fluorescence.

Unfortunately, using tryptophan is far from ideal, as the investigator cannot look at distinct regions of the protein, and in vivo measurements are impossible owing to interference from the fluorescence of other proteins. To increase specificity, investigators tried labeling proteins with exogenous fluorophores that could attach to cysteine side chains. However, these suffer from the fact that the wavelengths of light they absorb and emit overlap with cellular autofluorescence or have other properties that substantially reduce their usefulness in detecting conformational changes.

Around a quarter century ago chemists found that placing electron-donating and -accepting groups at opposite ends of an aromatic ring system produces very environmentally sensitive, or 'flaky', flurophophores. J an and colleagues have used 
receptor CCR5. Wolkowicz thus adopted this seven-transmembrane-domain protein as a scaffold and engineered libraries in which peptides are displayed in its aminoterminal region, which is exposed on the outside of the cells. Having learned from the lab's experience with peptide libraries, Wolkowicz made sure to constrain the peptides' structure to a loop conformation to increase the chances that peptides would retain their binding affinity once cleaved from the display scaffold.

The CCR5-based library, once stably expressed on the surface of human $\mathrm{T}$ cells through retroviral transduction, can be screened for binding to a 'bait' by flow cytometry. A proof-of-principle experiment showed that a peptide diluted in the library to a ratio of 1 in 10 million random clones could be recovered after only three rounds of FACS sorting with a high-affinity antibody.

Nolan explains that the choice of CCR5 ‥ was also influenced by the fact that it displays peptides extremely close to the membrane. This feature opens the possibility of using this system for finding reagents that have to be active in the context of the cell membrane, such as ligands, receptor decoys and antibodies that block viral infection.

As Nolan points out, it is also a way of making 'epitope mimics', or mimetopes, of surface proteins, and this is actually one of the reasons why the technique was developed in the first place. Nolan describes a particular monoclonal antibody directed against the HIV envelope that is broadly neutralizing, but whose epitope has been impossible to map. Mammalian surface display offers a way of using this existing antibody to screen a library of peptides in search of a mimetope that recapitulates the shape and immunoreactivity of the original epitope and that could be used to make a vaccine. "That was sort of the genesis of the original technique," says Nolan, "and now we are moving it forward. The next stage will actually be to put peptide libraries on and then go back to that original antibody."

\section{Veronique Kiermer}

\section{RESEARCH PAPERS}

Wolkowicz, R. et al. A random peptide library fused to CCR5 for selection of mimetopes expressed on the mammalian cell surface via retroviral vectors. J. Biol. Chem., published online on $18 \mathrm{~J}$ anuary 2005.

this pioneering work to create an improved fluorophore for labeling cysteine side chains on proteins (Cohen et al., 2005). This new probe, APM, is smaller and has a shorter linkage than many other fluorophores, meaning that there should be a smaller chance of its disturbing a labeled protein and less protein-independent movement. APM also has improved fluorescence properties, including excitation and emission spectra that are substantially different from most cellular autofluorescence.

The researchers tested the new probe by labeling sites on a potassium channel expressed in oocytes and a purified G protein-coupled receptor (GPCR). By mutating a desired site to a cysteine and mutating other accessible cysteine residues to serine, they were able to restrict labeling to specific sites. When they applied a voltage across the cell membrane containing the labeled channels, the resulting channel opening produced distinct changes in fluorescence that were proportional to the voltage applied. Similarly, the labeled GPCRs showed agonist-dependent changes in fluorescence that differed depending on the location of the label. Although the magnitude of the changes was not as great as that observed by using a FRET-based method to monitor conformational changes in GPCRs (Hoffman \& al., 2005) and the measurements were not obtained in live cells, APM could be used to look at region-specific conformational changes in receptors and is likely to be a valuable tool for examining conformational changes in diverse proteins.

\section{Daniel Evanko}

\section{RESEARCH PAPERS}

Cohen, B.E. et al. A fluorescent probe designed for studying protein conformational change. Proc. Natl. Acad. Sci. USA 102, 965-970 (2005).

Hoffman, C. et al. A FIAsH-based FRET approach to determine G protein-coupled receptor activation in living cells. Nat. Methods 2, 171-176 (2005). 